\title{
The Lung Mass and Nodule: A Case Series
}

Azam Jan', Naseer Ahmed²*, Nabil Iftikhar Awan', Bahauddin Khan³, Mujahid-ul-Islam³, Imtiaz Ahmed, Ahsan Shabbir ${ }^{3}$, Amir Mohammad ${ }^{1}$ and Hussain Shah ${ }^{1}$

'Department of Cardiothoracic Surgery, Rehman Medical Institute, Peshawar, KPK, Pakistan

${ }^{2}$ Institute of Basic Medical Sciences, Khyber Medical University, Peshawar, KPK, Pakistan

${ }^{3}$ Department of Cardiothoracic Anesthesia, Rehman Medical Institute, Peshawar, KPK, Pakistan

*dr.naseer99@gmail.com

\section{Abstract:}

Lung mass is an abnormal region of $3 \mathrm{~cm}$ or more in size present in the lungs mainly due to underlying pulmonary caner. It is usually round, opaque and poorly differentiated on X-ray. Common etiological key players are smoking, exposure to asbestos, radon, however, familial history may also play a role. We presented retrospectively 7 cases of lung mass and nodule encountered during our clinical practice. We have discussed their clinical presentation, manifestation, medical history, radiological findings and differential diagnosis. In this case series, most of the patients were young, only 2 cases were older patients. There was one infant one month old, one female child 12 years old, one female 25 years, 2 males, 22 and 21 years, one male of 50 years and another male of 60 years age. Correct diagnosis on the basis of clinical profile, radiological findings and histology may help in proper management and hence, timely treatment of the patient.

\section{Introduction:}

A "lung mass" is defined as an abnormal spot or area in the lungs that is more than $3 \mathrm{~cm}\left(1^{1 / 2}\right.$ inch) in size [1]. The commonest cause of a pulmonary mass is lung cancer. Risk factors for the lung cancers include; Tobacco smoke, Exposure to radon, According to US Environmental Protection Agency (EPA) report, Radon is the leading cause of lung cancer in non-smokers and is the second leading cause of lung cancers in smokers, Exposure to asbestos, Asbestos also have a greater risk of developing mesothelioma, other factors include prior radiation, genetics, and first degree relatives. The solitary pulmonary nodule is a rounded opacity, well or poorly defined on a conventional radiograph, measuring up to $3 \mathrm{~cm}$ in diameter. Opacity less than $3 \mathrm{~mm}$ is defined as a micro nodule. On CT scan 1-2 mm nodules are discovered in approximately half of the smokers aged 50 years or more. The frequency of SPN is variable and depends on the endemicity of granulomatous disease. Incidentally on imaging studies unrelated to the respiratory system in $0.09-0.2 \%$ of all chest radiographs [2]. Different characteristics that help us in discriminating between benign and malignant nodules are, Fat within an SPN is a reliable sign of benignity (hamartoma, lipoid pneumonia, and lipoma), and Bronchial carcinomas generally have a doubling time of 1 and 18 months. Furthermore, the short doubling time is another criteria for diagnosing malignant cancer. Another important factor is the location of the nodule. Most of the primary malignant nodules are located in the upper lobes, specifically on the right side, while two-thirds of metastatic pulmonary nodules affect the lower lobes [2]. Simultaneously the type of margins is another important criterion for diagnosing malignancy. Most malignant nodules present with irregular speculated and ill-defined margins. The most important factor in the assessment of cavitation within a lung mass is the wall thickness. Following is a retrospective review of cases we encountered in our clinical practice in which we had a suspicion of a lung mass or nodules on initial radiological assessment. We have ethical review board approval and consent of all the patients for this case series. The salient features are summarized in the table 1 and followed by details of imaging and discussion. 


\begin{tabular}{|c|c|c|c|}
\hline Case No. & Presentation & CXR findings & Diagnosis \\
\hline Case 1 & Dyspnea, fatigue & Lung nodules & Metastatic lung cancer \\
\hline Case 2 & Cough ,dyspnea & Well defined round mass & Hydatid cyst \\
\hline Case 3 & Fever \& productive cough & Complete left lung opacified & Bronchiectasis \\
\hline Case 4 & Severe respiratory distress & $\begin{array}{l}\text { Right upper lobe } \\
\text { consolidation }\end{array}$ & $\begin{array}{l}\text { Right middle lobe } \\
\text { emphysema }\end{array}$ \\
\hline Case 5 & Chronic productive cough & Shadow behind the heart & $\begin{array}{l}\text { Left lower lobe } \\
\text { bronchiectasis }\end{array}$ \\
\hline Case 6 & Cough & Left lower lobe mass & Pulmonary artery aneurysm \\
\hline Case 7 & Left chest pain & Left lower mass & Spindle cell tumor of lung \\
\hline Case 8 & Dyspnea, chest pain & $\begin{array}{l}\text { Multiple round bilateral lung } \\
\text { masses }\end{array}$ & Metastatic osteosarcoma \\
\hline
\end{tabular}

Table 1: Clinical presentation of cases

\section{Case 1:}

A 22 years old male patient presented to us who had a one-month history of fatigability progression of dyspnea on activity. No weight loss observed in the recent past, he was a tall man having 10 months history of house construction otherwise goes to school. He presented with decreased breath sounds towards the right side. No significant past history of any medical issues. On his first $X$-ray, there was unilateral pleural effusion (Figure $1 A$ ), which was drained but the lung did not expand completely (Figure 1B).

The fluid was tested showing hemorrhagic fluid cytology revealed atypical epithelial cells. We performed CT-scan which showed right lower lobe nodules and left lower lobe nodules (Figure $1 C, D)$. Diagnosis of metastatic lung tumor was made after biopsy with unknown primary site.

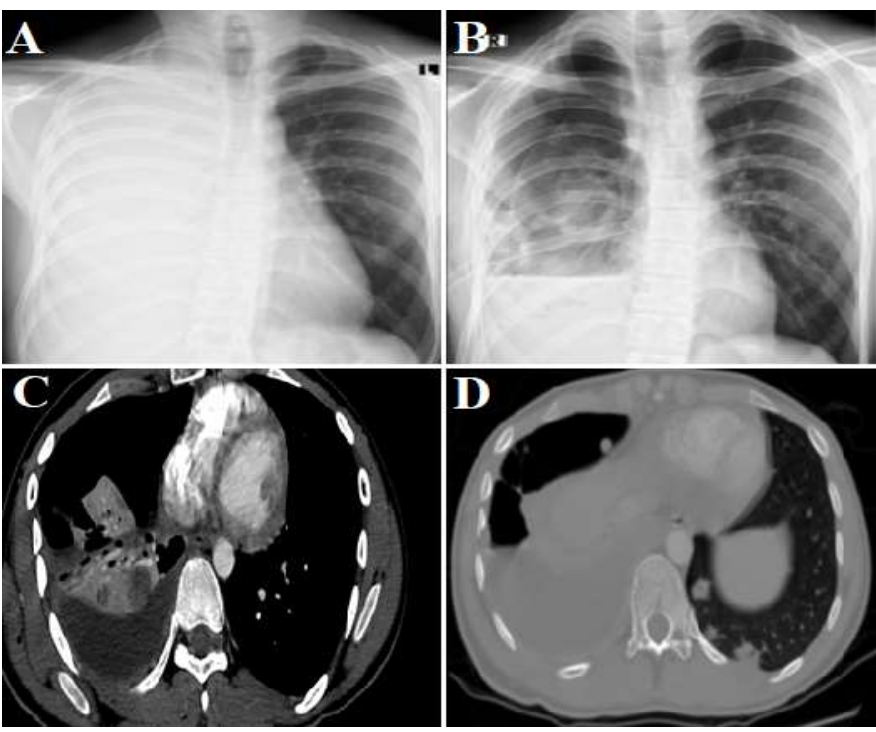

Figure 1A: Right thorax opacified completely because of unilateral pleural effusion B: Lung partially collapsed with left pleural effusion C: Right lung nodules/metastasis D: CT scan showing left lower lobe multiple lung nodules because of metastatic disease 


\section{Case 2:}

A 21 years old male presented to us with a cough, mild dyspnea but symptoms are worsening day by day. He was immigrant from Afghanistan. His chest $X$-ray showed large mass with smooth margins (Figure $2 A$ ). We did a CT-scan to confirm the diagnosis made on clinical suspicion i.e. hydatid cyst (Figure 2B, C). Patient was operated successfully and cyst was removed (Figure 2C, D).
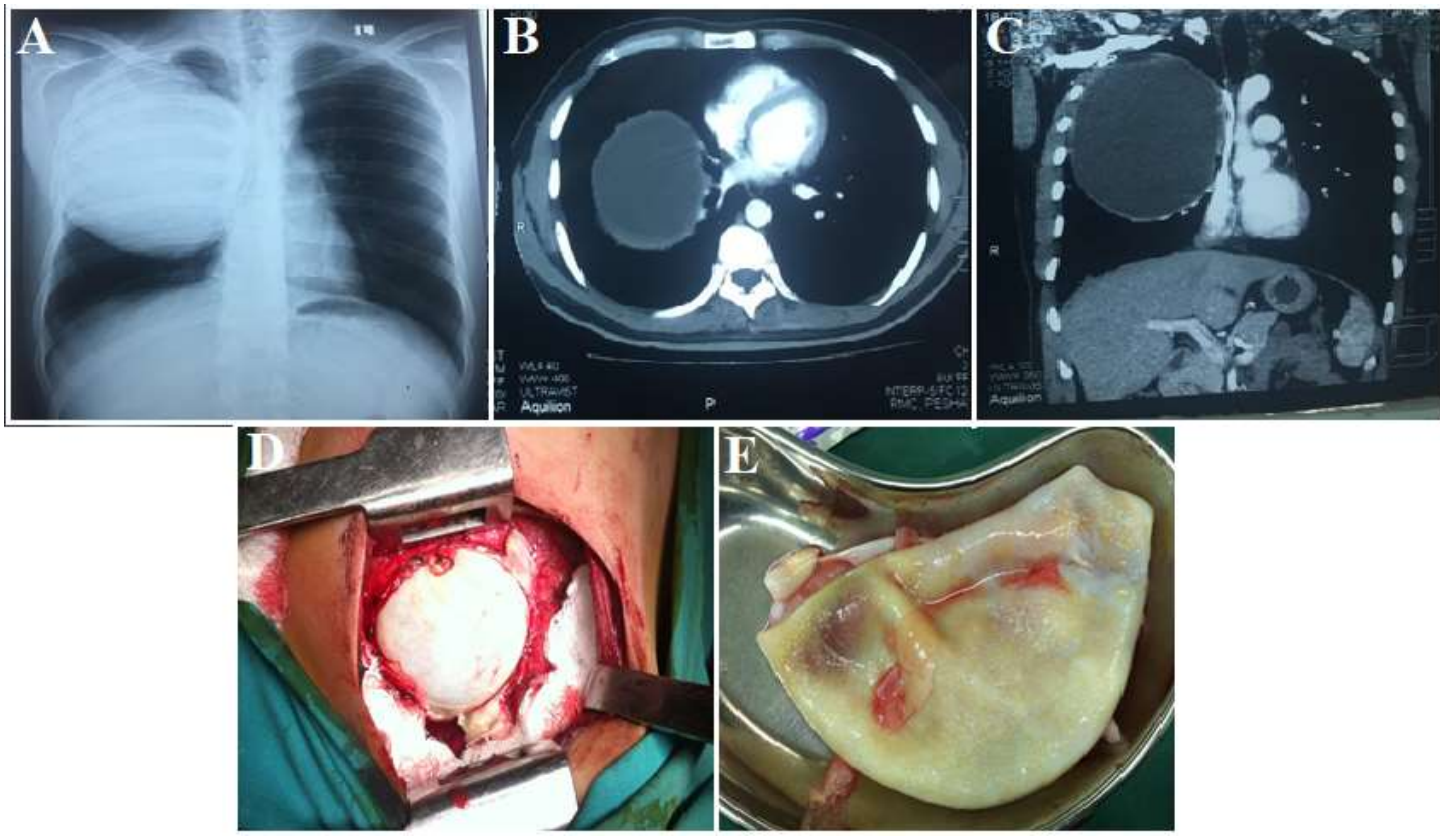

Figure 2A: Right lung showing cystic opacity B, C: CT scan shows right sided cystic mass D: Hydatid cyst protruding from the lung $\mathrm{E}$ : hydatid cyst after removal

\section{Case 3:}

A 12 years old young female patient, presented to us as pale looking, accompanied with fever and shortness of breath. Previously she had a chronic cough but now this time it was a productive cough. Chest X-ray demonstrated opacification on left side of chest (Figure 3A). CT-scan was very helpful to give us a diagnosis of bronchiectasis (Figure 3B,C). Diagnosis was Pediatric bronchiectasis. Cystic fibrosis related Bronchiectasis and Non-cystic Fibrosis Bronchiectasis. In symptomatic patient with good pulmonary reserve surgery is mandatory.
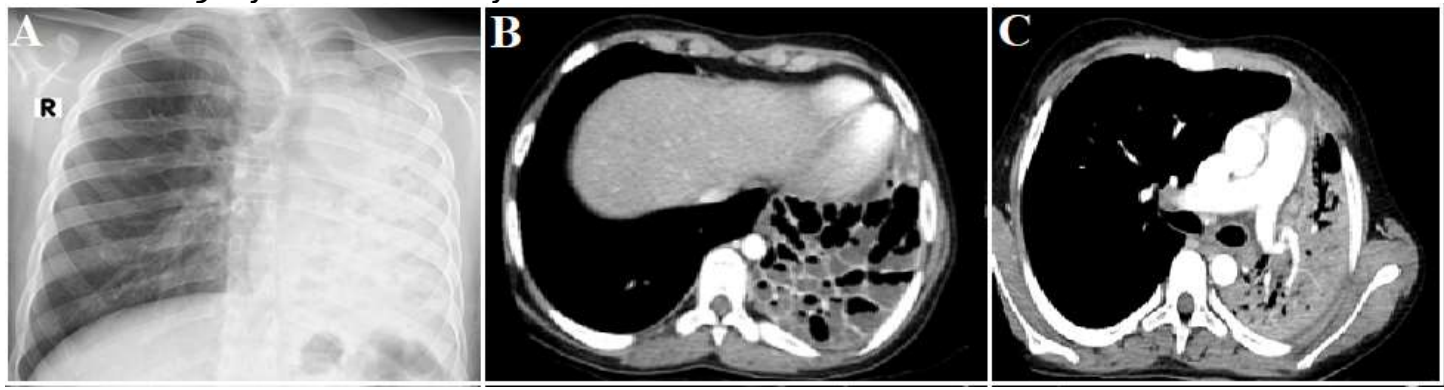

Figure 3A: Left side thorax opacified with air Broncho-gram

$B, C$ : $C T$ chest with contrast shows left sided diffused bronchiectasis 
Case: 4.

A one month old baby, admitted to NICU with complaints of severe respiratory distress, started few days ago and now worsening of sign and symptoms. No birth problems. Bilateral breath sounds were present. On chest $X$-ray right upper lobe consolidation and lower lobe over expansion was seen (Figure 4A). Patient was urgently intubated and CT-scan was done. Diagnosis was congenital lobar emphysema of the right middle lobe (Figure 4B). Right middle lobe lobectomy was done

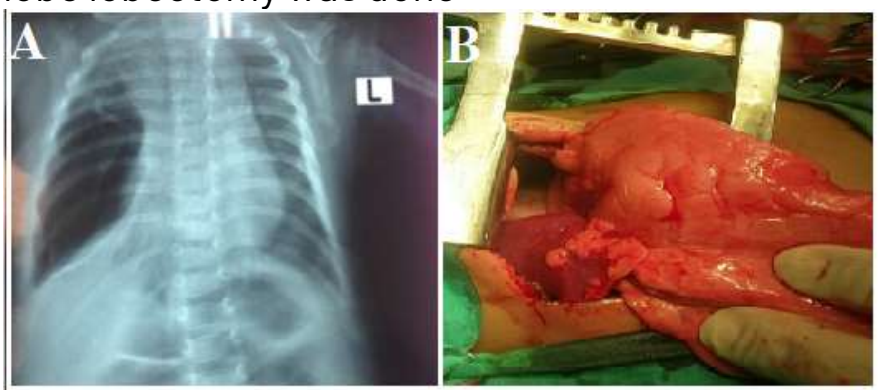

Figure 4A: Right upper lobe consolidation \& Right lower lung over expansion B: Emphysema of the right middle lobe

\section{Case 5:}

A young female patient, presented with chronic cough with sputum, Episodes of fever on/off. She had been on multiple long term antibiotic therapies and had seen by multiple physicians. She underwent multiple chest $\mathrm{x}$-rays in the past and was considered normal. Recent $X$-ray shows abnormality behind heart at left lung (Figure 5 ).

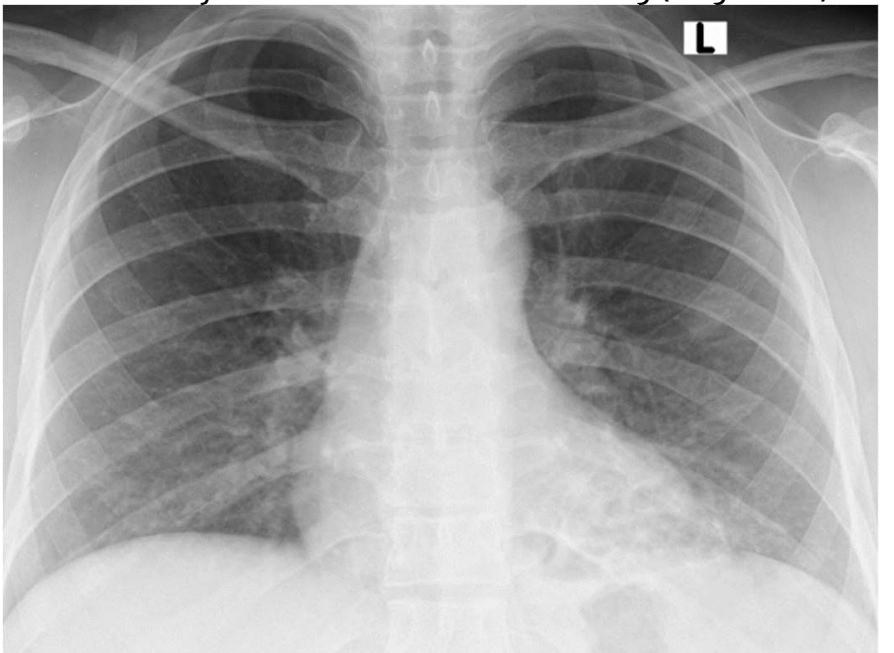

Figure 5: Abnormal left lung behind the heart, Dx: Bronchiectasis (adult) of left lower lobe, patient underwent left lower lobectomy
Case 6:

A 50 years old male patient, who was presented to us with complaints of cough, un-controlled diabetes. There was no history of fever and tuberculosis. No previous imaging was found. He has used cough suppressant medications as an outpatient. On chest $\mathrm{x}$-rays left lower thorax opacity was observed (Figure 6). Final histopathology report Mucormycosis which had caused this problem.

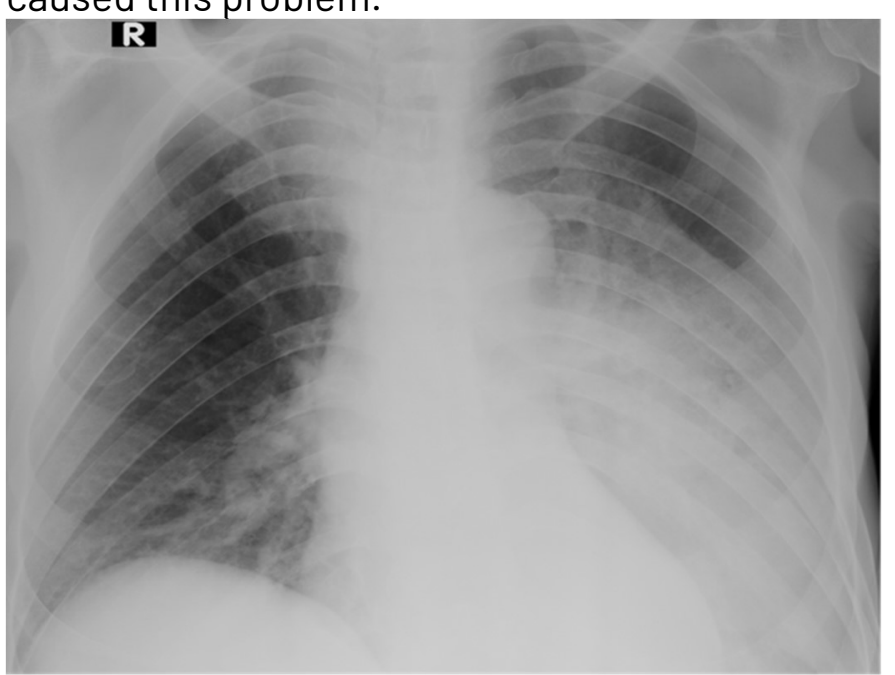

Figure 6: Left lower thorax opacity, Dx: Mucormycosis of lung/Fungal infection

\section{Case 7:}

A 60 years old male patient, presented to us with mild left chest pain, very active. On further questioning some sort of decrease in activity because of feeling tired, No weight loss observed, No change in appetite was observed. There wasn't any history of cancer. Chest X-rays of this patient also shown left lower thorax opacity (Figure 7). We did CT guided biopsy and mass showed spindle cell tumor (Figure 7).

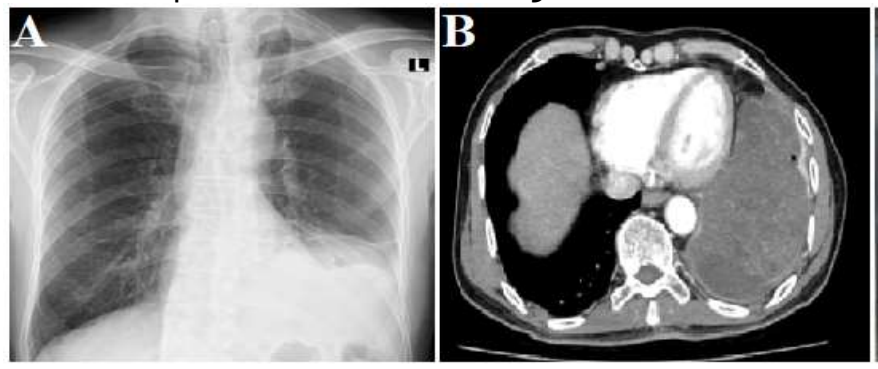

Figure 7A: Left lower thorax opacity B: CT chest shows left lower mass compressing the lung, Dx: Spindle cell tumor of the lower chest. Excision of the tumor and left lower lobectomy done 


\section{Case 8:}

A 25 years old young female patient, diagnosed case of Osteosarcoma of left knee joint, and tumor resection surgery was done 6-months back. Recently she presented with shortness of breath, fever on/off, Productive cough, Chest discomfort and his chest X-rays demonstrated bilaterally multiple circular masses(Figure 8 )

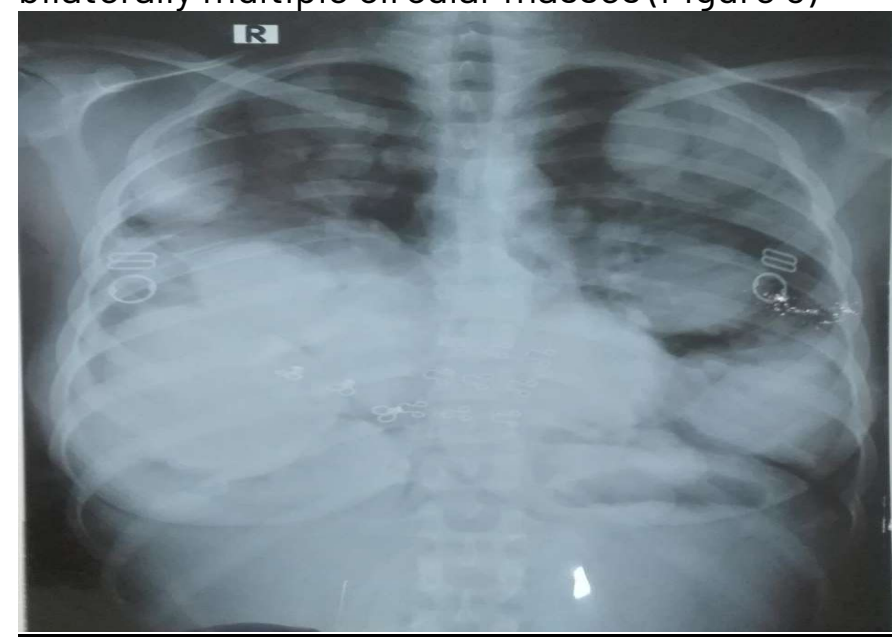

Figure 8: Multiple circular masses in bilateral lungs,

Dx: Osteosarcoma of left proximal tibia with metastatic disease to the lung

\section{Discussion:}

Lung cancers are the leading cause of deaths worldwide among cancer-related deaths. With non-small cell lung cancer accounting for around $85 \%$ of all deaths due to lung cancers. Metastatic non-small cell lung cancer is rare in patients under 25 years of age. Other malignancies that can occur in this age group are germ cell tumors (teratocarcinoma), lymphoma, carcinoid and metastasis from a non- lung primary cancer [3]. At presentation, around $30 \%$ of the NSCLC are localized and surgery is the treatment of choice for the disease. However, in cases of successful surgeries, recurrences are seen in most of the patients as the disease takes its course. For those patients who are inoperable with locally advanced disease or metastatic disease at the time of diagnosis, there are no curative treatments available. As a whole, the prognosis of lung cancers is bad with a less than $15 \%$ of 5 year survival rate [4].

Infections of lung are very common problems.Infections can be bacterial, viral,parasitic or fungal.The most common parasitic infection requiring surgery is Hydatid cyst. Hydatid disease is a parasitic infestation caused by Echinococcus granulosus. Majority of lung hydatid cysts are silent and either small or medium in size. The non-complicated hydatid cysts are usually found accidentally during routine chest $x$ - rays for other than chest diseases [5]. The Giant hydatid cysts and complicated cysts are symptomatic that includes compression symptoms i.e. a dry cough in case of large cysts, and productive cough in cases of communication with the bronchial tree. Other symptoms include chest pain and dyspnea in case of a ruptured pleural cavity [6].

In cases of complicated hydatid cysts, CT scan has shown to be an important imaging modality. Various clinical signs that are associated with hydatid cysts includes "Serpent sign" and "Spin sign" indicates a collapsed parasitic membrane. Other classical signs include "Signet ring", "water lily", "Crescent or meniscus", "inverse crescent", air bubble and dry cyst [7]. In lungs, $72 \%$ of hydatid cyst involve one lobe, usually the lung bases. But if the cyst ruptures than a radiological image of pneumopericyst are seen. If the content of cyst is completely evacuated to the bronchial tree, then a similar cavity to tuberculosis or pulmonary abscess appears. Bilateral hydatid cyst disease of the lungs may be managed by one or two-stage surgery i.e. bilateral thoracotomy, VATS etc. Medical Therapy of inoperable cysts with albendazole has produced improvements [8].

Extensive clinical, labs, and pathological investigations up to $50 \%-80 \%$ of cases are still idiopathic. Infections that occur in childhood including pertussis were thought to have $11 \%$ of bronchiectasis cases and $10 \%$ cases are related to prior granulomatous diseases [9]. It is not important for a patient with bronchiectasis to have normal chest radiographs, in fact, serial radiographs allow identification of progressive disease. A congenital form of obstructive lung disease can present at birth or early age and can be life threatening.Congenital lobar emphysema 
(CLE) is a rare abnormality of the lung development which can cause respiratory insufficiency of a suckling child. It is caused by the hyperinflation of the lung lobe with compression of the normal lung parenchyma and contralateral displacement of the mediastinum [10]. Although bilateral involvement has also been reported. In our case Right middle lobe was involved [11].

Most of the time it is presented in its acute phase whereas chronic presentation is rarely seen. Lee et al. [14] did a retrospective review of mucormycosis and demonstrated that diabetes mellitus was the most common risk factor, among 86 cases $56 \%$ patients were diabetic. There are many cases reported from India suggesting that pulmonary mucormycosis mimics tuberculosis (TB), sometimes nonresolving pneumonia or a co-infection with TB [12-13]. The sign and symptoms of pulmonary mucormycosis are non-specific and it is difficult to distinguish it from other fungal infections like aspergillosis on chest $\mathrm{X}$-rays. It may present as lungs cavitation, lobar consolidation, nodular infiltrates, lung mass or an infarct due to pulmonary vessel thrombosis by fungal angioinvasion. "Halo sign", "reversed halo sign" may be correlated with massive hemoptysis [14]. Diabetic patients are more at a risk of developing the endo-bronchial disease [15]. Our patient presented with sign and symptoms that mimic tuberculosis, so he was diagnosed as a case of TB outside and was started with anti-tubercular drugs. The outcomes and prognosis of pulmonary mucormycosis have improved in the last few years due to early diagnosis of the disease, surgical debridement, and latest antifungal agents. The most recommended agent these days is liposomal amphotericin B, but concerns regarding limited penetration of disease site by this drugs are also there. Several studies have been done to study the combination of early surgical resection with antifungal cover and it has shown significant improvement in survival of the patients [16].
The major causes of pulmonary artery aneurysm include congenital and acquired including untreated syphilis and tuberculosis, Vasculitis of the pulmonary artery, Pulmonary Artery hypertension (PAH), Chronic pulmonary embolism [17]. According to the WHO histological classification 2015, pulmonary Sarcomatoid carcinoma (PSC) includes pleomorphic carcinoma, giant cell carcinoma, carcinosarcoma and pulmonary blastoma. All of them are having a low incidence and specifically, PSCC is very rare, accounting for only $0.2 \%$ to $0.3 \%$ of all lung cancers [18].

Spindle cell sarcoma begins as a small lump in stage 1. At this stage, it can be excised with a rim of healthy tissue and this stage has a good prognosis as well. If it is left untreated and progress to stage II or III, then micro metastasis occurred can be hardly treated even if the lump is excised. Complete cure is then not possible. However, chemotherapy or radiotherapy is required for stage II and III sarcomas [19].

The lung is the most common site of for metastasis of osteosarcoma [20]. Osteosarcomas have a dramatic response to treatment, $25-30 \%$ of the patients eventually develop metastasis. Approximately one-third of patients having osteosarcoma will experience a recurrence of the disease, $95 \%$ within 5 years of initial diagnosis. Average survival after recurrence is less than 1 year. Patients in a second remission have a $70 \%$ to $80 \%$ chance of the second relapse within 1 year [21].One rare cancer of lung is spindle cell carcinoma. Spindle cell tumor (Pulmonary spindle cell carcinoma: PSCC) is a high-grade malignant tumor of the lungs, also called "Sarcomatoid carcinoma (SC)" or "pseudosarcoma".

\section{References:}

1. https://www.verywell.com/lung-masspossible-causes-and-what-to-expect2249388

2. Khan $\mathrm{AN}, \mathrm{Al}$-Jahdali $\mathrm{HH}$, Irion $\mathrm{KL}$, Arabi $M$, and Koteyar SS (2011). Solitary pulmonary nodule: A diagnostic algorithm in the light of 
current imaging technique. Avicenna $J$ Med. 1(2): 39-51.

3. Jemal $A$, Siegal $R$, Ward E, Hao $Y, X u$ J, Thun MJ (2009). Cancer statistics, 2009. CA Cancer J. Clin. 59:225-49.

4. Ki-Rok Kwon1, Hyundo Kim, Jung-Sun Kim, Hwa-Seung Yoo, Chong-Kwan Cho (2011). case series of non-small cell lung cancer treated with mountain ginseng pharmacopuncture. J. Acupunct. Meridian Stud., 4(1):61-68.

5. S.M. Sadjjadi (2006). Present situation of echinococcosis in the Middle East and Arabic North Africa. Parasitol. Int. 55.197e202.

6. Robert ES, Eugene JM, William FM, Sally HE, Stacey M (1999). Case records of the Massachusetts General Hospital. Weekly clinicopathological exercises. Case 291999. A 34-year-old woman with one cystic lesion in each lung. $N$ Engl. J. Med. 341(13): 974-982.

7. Engstrom ELS, Salih GN, Wiese L(2017). Seronegative, complicated hydatid cyst of the lung: A case report. Respiratory Medicine Case Reports, 21,96e98.

8. Pedro M, Schantz M (2009). Echinococcosis a review. International Journal of Infectious diseases, 13:125-133.

9. Metersky ML (2010). New treatment options for bronchiectasis. Ther. Adv. Respir. Dis., 4:93-9.

10. O'Donnell AE (2008). Bronchiectasis. Chest, 134:815-23.

11. Abdellah 0 , Mohamed $\quad H$, Youssef B, Abdelhak B (2013). A Case of Congenital Lobar Emphysema in the Middle Lobe, J. Clin. Neonatol. 2(3): 135-137.

12. Ringshausen FC, de Roux $A$, Diel R, Hohmann D, Welte $T$ and Rademacher $J$ (2015). Bronchiectasis in Germany: a population-based estimation of disease prevalence. Eur. Respir. J. 46(6):1805-7.

13. Garg R, Marak RS, Verma SK, et al., (2008). Pulmonary mucormycosis mimicking as pulmonary tuberculosis: a case report. Lung India, 25:129-31.

14. Lee FY, Mossad SB, Adal KA (1999). Pulmonary mucormycosis: the last 30 years. Arch Intern Med, 159:1301-9.

15. Iqbal N, Irfan M, Jabeen K, Kazmi MM, Tariq MU, (2017). Chronic pulmonary mucormycosis: an emerging fungal infection in diabetes mellitus. J. Thorac. Dis. 9(2):E121-E125

16. Hamillos G, Samonis G, kontoyiannis DP (2011). Pulmonary mucormycosis. Semin. Respir. Crit. Care Med. 32(6):693-702.

17. Kreibich $M$, Siepe $M$, Kroll J, Hohn R, Grohmann J, Beyersdorf F (2015). Aneurysms of the pulmonary artery. Circulation. 131:310-316.

18. Dian-Jun Oi, MD, Bing Liu, MD, Liang Feng, MD, Lin Zhao, MBBS, Ping Yan, MBB, Jiang Du, MD, Oing-Fu Zhang, MD Pulmonary spindle cell carcinoma with unusual morphology Medicine (2017) 96:24(e7129).

19. Aliena Badshah, Salman Khan and Usman Saeed Spindle Cell Sarcoma Presenting as Pancoast Syndrome JPMA 2016, Vol. 26 (7): 623-625.

20. Vander Griend RA (1996). Osteosarcoma and its variants. Orthop. Clin. North Am. 27:57581.

21. Kempf-Bielack $B$, Bielack SS, Jürgens $H$, Branscheid D, Berdel WE, Exner GU et al., (2005): Osteosarcoma relapse after combined modality therapy: An analysis of unselected patients in the cooperative osteosarcoma study group (COSS). J. Clin. Oncol, 23:559-568. 